\title{
The Power of Assumptions
}

\author{
Yongchen Zou \\ School of Finance and Statistics, East China Normal University, Shanghai, China \\ E-mail: rustyzou@gmail.com
}

Received June 25, 2011; revised July 28, 2011; accepted August 8, 2011

\begin{abstract}
This paper examines computational merits provided by assumptions made in scientific modeling, especially regression, by trying to exhibit abstractly a model deprived of those assumptions. It shows that the principle of Occam's Razor has been mistakenly used as model developers' justification to keep scientific models "as simple as possible", and that the cost of inflating computability is truncation of model robustness.
\end{abstract}

Keywords: Scientific Modeling, Simple Regression Model, Induction, Naïve Generalization

\section{Introduction}

It is impossible to prove right of things without assuming some others true. But more assumptions mean necessarily more chinks in a certain theory for it might collapse as long as any one of the assumptions is demonstrated invalid. In this paper I attempt to examine computational merits provided by assumptions made in scientific modeling, especially regression, by trying to exhibit abstractly a model deprived of those assumptions. It is shown that the principle of Occam's Razor has been mistakenly used as model developers' justification to keep scientific models "as simple as possible", and that the cost of inflating computability is truncation of model robustness.

The rest of the paper is organized as follows: Part I discussed basically the validity and applicability of scientific modeling and particularly, the method of regression. Part II poses criticism of simple regression theory insofar as its understanding of randomness. Part III shows naïve inductive generalization in regression model and proposes a de-generalized model to manifest power of assumptions. The last section of Part III is a brief conclusion.

\section{Validity and Applicability of Scientific Modeling}

\subsection{From Observations to Theories}

Popper [1], [2] wrote in his criticism of "anti-naturalistic" doctrines of historicists that theories manifest priority to observations and experiments for it is theories that make empirical or experimental evidences relevant. He criticized the method of generalization in its presuming science to be developed through deriving theories from observations. However, Popper does not deny the coexistence of theorization and observation; in other words, the criticism is an argument for philosophical order, that theorization calls for observing rather than the reverse, and it does not suggest exclusiveness between the former and the latter. As a matter of fact, it is unobjectionable that a theory is not validated before tested by observation and experiment, and observations and results of experiments would be of greater value when serving as evidences of a certain theory.

It seems, however, difficult to discern philosophical order of observation and theories in scientific modeling. It can be that the practice of modeling, a sole part of theorization, is inspired by an unintended observation from nature or laboratory. History is rich in examples of serendipitous findings resulting in scientific break-through. But also common are the cases where data are collected with respect to what is required in testing $a$ priori, or a supposed model usually with parameters to be estimated. And one should also realize that, more often than not, theorization and observation are prompted reflexively. We might get intrigued by a certain phenomenon, which evolved into our raw interests in modeling, thus explaining, it, but the very impetus wouldn't have been there if it was not for data we randomly observed in the first place. On top of that is the polemic of validity of mathematical modeling, which in language of mathematics attempts to explain and predict behaviors of natural or human systems of various, if not all, kinds. Albeit a crowning field that compels academic contributions, mathematical modeling and its derivative braches, nota- 
bly financial modeling, have received wide suspicions for its vulnerability towards the tests of reality, especially through crises. And it is even pointed out by Taleb [3] a hubristic side effects, or a Procrustes problem, of modern civilization that reality is ludicrously blamed for not fitting scientific models.

When a method errs, it is either because it was born logically erroneous, i.e. in our case the mistaken philosophical order concerning theories and observations, or it is, in the sense of methodology, designed inappropriately. We have shown so far that it would be more or less futile to rigidly make clear the order of theories and observation, and this essay centers therefore its considerations solely on the methodology. I shall speak of the regression model, which is to be the subject of this essay. But before that I am obliged to give a word on a frequently referred principle in scientific modeling termed Occam's razor.

\subsection{Occam's Razor}

The principle of William of Ockham suggests that entities must not be multiplied beyond necessities. And it calls for competing theories and hypotheses that presumed the least. The metaphor "razor" stems from its core argument of shaving away redundant assumptions. This doctrine of simplifying things as densely as possible seems to touch well upon the idea of scientific modeling of any kind, but willy-nilly interpreted as we should keep models as simple as possible. As a matter of fact, it has even been misleadingly used as justification for many otherwise obviously invalid assumptions made only for computational or analytical merits. For example, econometricians believe their theories to have been endorsed by the Razor in keeping regression models as simple as they could be, so their weapons of calculus and statistical inference could enter the picture. Gujarati and Porter [4] suggested further that it is in the light of Occam's Razor that combined impact on explained variable of factors other than the assumed explanatory variable(s) can be viewed non-systemic. And it is under this critical assumption that the error term $\varepsilon$ is introduced as normally distributed so that we gain the power to deal with the unobservable. I will explore the chinks in basic idea of oversimplified regression model although it should be recognized that my analysis does not indicate embrace of model complexity. Rather, it aims at showing what has been provided by assumptions in regressive modeling, and the method employed in generalization and induction.

\subsection{Regression Analysis of Nature and Society}

It seemed once reasonable, and it still is, believed some- one, to nurse the belief that there should be a fine line between applicability of mathematical and physical theories in natural and human phenomena. It was argued that what functioned well in natural science are buttressed in their validity by the relatively stable properties of natural objects in their reaction to the changes, say, of circumstances, while human beings, both from collective and individual perspective, manifest nature of volatility and autocorrelation, in the sense of, for instance, herd behavior, toward daily encountering.

Plausible as it may sound, drawing a line between natural and human phenomena seems all the more a Utopian proposal, perhaps thanks to social dynamic, for there emerged institutions who play irregularly in domains not able to be identified clearly "natural" or "human". For example, a market player who invested his funds in SPDR Gold Trust would find himself involved in social events, e.g. fluctuation of dollar, change in interest rate, other market players' psychology, potential demand for the bullion from developing economies, as well as influenced by natural factors, e.g. change of supplies of gold and other precious metal, momentary collapse of confidence incurred by certain natural catastrophes. It seems therefore impossible to distinguish applicability of theories in nature and in society where, especially in financial markets, natural and human forces that lead to occurrences of certain events are themselves inseparable. One of the major characteristics of the method of regression, and also one of the reasons it is chosen as representative of scientific modeling, is that it attempts to reveal impact that factors cast upon other factors. In a simple model of regression, an explanatory variable $X_{i}$ is said to be responsible for behavior of explained variable. But it is usually overlooked, especially in a system of complexity, that $X_{i}$ would itself be correlated with other factors so that they would cast a combined influence on the explained variable. That is, alternatively, the explanatory variable is a variable conditional on other correlated ones expressed probably as $X_{i} \mid X_{j}=x_{j}$.

It should be noted that epistemological problem of method of regression does not confine to its applicability. For example, Robinson [5], Goodman [6], and Lichtman [7] examined the collective-to-individual "cross-level" inference of regression in sociological theories. Kydland and Prescott [8], Smets and Wouters [9], Sims [10] contributed to richer interpretations of non-experimental inferences of method of econometrics.

\section{Error Term the Pseudo-Randomness}

\subsection{Criticism of Error Term of Simple Regression Model}

According to the simple regression model, the explained 
variable $Y$ is expressed as a linear function of explanatory $X$ by

$$
Y=b_{0}+b_{1} X+\varepsilon
$$

where $b_{0}$ is the intercept parameter and $b_{1}$ the slope parameter measuring sensitiveness of behavior of $Y$ with accordance to that of $X . \varepsilon$ is the error term responsible for any factors other than $X$ that affects $Y$ under the assumption that $\varepsilon$ is normally distributed with mean zero or

$$
E(\varepsilon)=0
$$

In practice the only work for the model assumer is to have the two parameters well estimated, under observations of $\left(Y_{i}, X_{i}\right)$, with mathematical technique termed Least Squares (LS), given by the equations

$$
\begin{gathered}
b_{0}=\frac{1}{n} \sum_{i=1}^{n} Y_{i}-\frac{\sum_{i=1}^{n} X_{i} \sum_{i=1}^{n}\left(X_{i}-\frac{1}{n} \sum_{i=1}^{n} X_{i}\right)\left(Y_{i}-\frac{1}{n} \sum_{i=1}^{n} Y_{i}\right)}{n \sum_{i=1}^{n}\left\{\left(X_{i}-\frac{1}{n} \sum_{i=1}^{n} X_{i}\right)^{2}\right\}} \\
b_{1}=\frac{\sum_{i=1}^{n}\left(X_{i}-\frac{1}{n} \sum_{i=1}^{n} X_{i}\right)\left(Y_{i}-\frac{1}{n} \sum_{i=1}^{n} Y_{i}\right)}{\sum_{i=1}^{n}\left\{\left(X_{i}-\frac{1}{n} \sum_{i=1}^{n} X_{i}\right)^{2}\right\}}
\end{gathered}
$$

Subtract the error $\varepsilon$, which is a stochastic term, from equation (1) we get the regression function geometrically sketched as a line shaped with intercept $b_{0}$ and slope $b_{1}$. From equation (3) and (4) of Least Squares estimator technique it is shown that value of $b_{0}$ and $b_{1}$ are determined exclusively by observations $\left(Y_{i}, X_{i}\right)$. In other words, shape of the regression function is determined by data $\left(Y_{1}, X_{1}\right),\left(Y_{1}, X_{1}\right), \cdots,\left(Y_{i}, X_{i}\right)$. But one should be aware of the fact that estimates of intercept and slope parameters are tenable only for a given set of data. That is, so long as another arbitrary observation is added to the initial data, computational values of $b_{0}$ and $b_{1}$ should be revised to what is derived by equation (3) and (4) with $\left(Y_{1}, X_{1}\right),\left(Y_{1}, X_{1}\right), \cdots,\left(Y_{i}, X_{i}\right)$ substituted by $\left(Y_{1}, X_{1}\right),\left(Y_{1}, X_{1}\right), \cdots,\left(Y_{i}, X_{i}\right),\left(Y_{i+1}, X_{i+1}\right)$, and they are, except for some rare cases, very unlikely to assume the values they used to do.

I suggest what this means to the method of regression is ironical because a regression model is supposed to reflect the relationship between behaviors of explanatory and explained variables by means of determining $b_{0}$ and $b_{1}$. However, analysis above has just shown that the shape of the regression function is determined not by the true bond between $Y$ and $X$, but by the availability of data. In real studies, however, we are not always confident about neither reliability nor completeness of the data we obtain.

We may also look at what is said about the error term $\varepsilon$. It is also called "disturbance" of regression for it attempts to explain the non-systemic deviation of data from the regression line. But this terminology seems to contradict the definition, and I would attribute this error to the confounded understanding of concepts of functional-relationship model and regression model. A functional-relationship model is buttressed usually by axioms and ironclad theorems, whereas a regression model is backed by a theory far from being indubitably proved. Consider modeling the area of a given circle by

$$
S=\pi(R+\varepsilon)^{2}
$$

where $S$ denotes the area of the circle, $R$ is the observed radius and $\varepsilon$ the non-systemic error incurred by minor impacts of other factors like temperature and gauging error. In a functional-relationship model as this, the error term is justified to be termed "disturbance" for factors other than observed radius is proved negligibly weak in affecting the area. Yet when we think of a regression model that regresses height against weight of an individual, it is insecure to assert variables other than weight are of minor significance for unlike equation (5), functional-relationship between height and weight of human beings is far from a rigorously proved theorem.

Another tricky argument for normality of the error term is given by statistical advantage of the central limit theorem. Because there are too many factors affecting the explained variable, their mean impact is then according to central limit theorem asymptocally normally distributed. This is a misleading conclusion for first, the theorem can be proved tenable by using moment generating technique only for large number, but one in developing a regression model is not bestowed with the priori of exactly how much factors other than the arbitrarily assumed independent variable are affecting the dependent variable (otherwise those influences would be incorporated into the model if one attempts to keep the model reasonable and efficient), thus it is unjustifiable to assert other factors to be "too many". Second, central limit theorem is developed upon a sheer probabilistic and numerical premise. Random variables, whatever distribution they have, are of no superior significance to each other. In a large size sample composed of random variables of various distributions, one with normal distribution is of no greater impact than one with gamma. Central limit theorem in this sense is not significanceweighted. This is remarkably untrue for regression model in that real life factors are always in different superiority in explaining a certain end. For example, the depressive impact on stock prices of a natural disaster in the short run, we learned from history, is usually more eminent 
than that of a raised interest rate. In fact, the assumption of the error term given by equation (2), I believe, seems more like representative of a common belief in mean reversal, or embrace of Aristotelian natural places, that data, for the lone run, though deviate from the regression line, always show the "natural tendency" to return to normal.

Besides, from a behavioral aspect, people's understanding of error term, I believe, has been more or less manipulated by its denotation. Error term and regression residual, estimator of error term, are denoted either $\varepsilon_{i}$, $e_{i}$, or $u_{i}$, letters of minutest size that could ever be found, and small in size is inclined to be heuristically interpreted as minor in significance, at least inferior to variables expressed in capital letters $Y$ and $X$. I doubt that an equation of

$$
Y=b_{0}+b_{1} X+U
$$

merely with $u$ substituted by a capital $U$ would encourage more serious considerations on the error term, ludicrous as it might sound.

\subsection{Err Function}

It must be admitted that if we deny the normality of the error term, given the hitherto analysis, we lose the computational merits brought about by such approach. This section is to show abstractly the difficulty in revising the regression model entailed by "shaving off" the invalid assumptions about $\varepsilon$ and thus at the same time to reveal the power of these assumptions.

I have shown in the previous section that error of regression model comes from first, the inaccuracy and incompleteness of data, and second, other but not assumed as explanatory factor(s) that influence the dependent variable, both of which systemic. Error of first kind could be measured by a degree of unavailability of data on presumed dependent and independent variables, denoted $\theta$, as measuring systemic error, e.g. data manipulation and intransparency, in data fetching. While the second kind of error is expressed as a function of non-explanatory factor(s). Define an Err element and plug it into a simple regression model substituting error term $\varepsilon$

$$
Y=b_{0}+b_{1} X_{1}+\operatorname{Err}\left\{\theta ; X_{j,(j \neq 1)}\right\}
$$

in which $\theta$ is called the unavailability strength of data set $\left(Y_{i}, X_{1 i}\right), X_{j}$ are responsible factors that are not presumed by model developer as explanatory variables, and $\operatorname{Err}(\bullet)$ is a function of $X_{j}$ with parameter $\theta$.

Finitude of data, it shall be pointed out, must be examined before model (6) can be generalized into a multivariate version. However, regression model since invented has been built upon a stationary premise that technically it focuses on explaining relationship of explained and explanatory factors on the time point when the data is fetched. From a stationary point of view, data is hardly infinite. Hence intuitively value of $\theta$ would not be infinity and so is the case for numbers of factors $X_{j}$ that constitute second kind of error of the model. But this is would be a blunder if we assume a dynamic perspective, for not only there appear new data that was not available and factors that did not impact explained variable yesterday, but also there factors used to have a voice in determining $Y$ might today lose their forces and should be eradicated from the model.

Based on the stationary premise of regression model, we write model (6) into a multivariate version

$$
Y=b_{0}+\sum_{i=1}^{n} b_{i} X_{i}+\operatorname{Err}\left\{\theta ;\left\{X_{i}^{C}\right\}\right\}
$$

where $\left\{X_{i}^{C}\right\}$ is the complement of $\left\{X_{i}\right\}$ under the assumption of finite factors responsible for $Y$. Now other than estimating parameters $b_{0}, b_{1}, \cdots, b_{n}$, one needs an estimator of $\theta$, the unavailability strength. It has been shown that $\theta$, measure of the unavailability, is entailed systematically by imperfect data, thus it would be fallacious to simply resort it to randomness, although it seems at the first glance reasonable to do so. But to reject sacrificing model robustness for solvability raise, as is done by the common wisdom to stamp a normal distribution on disturbance, would be costing because paradoxically to estimate $\theta$ is to estimate how much you don't know, which seems to be epistemologically impossible.

Alternatively, we may decompose the unavailability strength into what it aims to express, namely the imperfect nature of data. Model (7) is then transformed into

$$
Y=b_{0}+\sum_{i=1}^{n} b_{i} X_{i}+\operatorname{Err}\left\{\left\{y_{i^{C}}, X_{i^{C}}\right\} ;\left\{X_{i}^{C}\right\}\right\}
$$

In model (8), strength of data unavailability $\theta$ is substituted by set $\left.\left\{y_{i c}, x_{i}\right\}\right\}$ which stands for complement of data sets $\left(Y_{i}, X_{i}\right)$, still assuming finite data source. This is the basic idea of developing robust regression model which values significance of model error as much as of presumed dependent and independent variables. The model separates itself into the observable and the unobserved, or the Err part. However, it will be further shown in the subsequent sections that even equation (8) is a somewhat fragile regression model due to mistakable generalization and induction.

\section{Naïve Inductive Generalization \& A De-Generalized Form of Regression Model}

\subsection{The Assumption of Addition}

Return to Occam's Razor discussed earlier; recall that it calls for assumption austerity but, I claimed, only to be 
falsely interpreted as "keep things as simple as possible". This can be seen in, for example, the very common practice that equates "and" to "add". In regressive modeling, if behavior of $X_{1}$ and $X_{2}$ are believed to be responsible for change in $Y$, then it is speculated that

$$
Y=f_{1}\left(X_{1}\right)+f_{2}\left(X_{2}\right)+\varepsilon
$$

Computational benefits allowed by addition are relentless. For example, one is free of the concern of commutativity that may otherwise engrained in exponentiation of model (9) for $f_{1}\left(X_{1}\right)+f_{2}\left(X_{2}\right) \equiv f_{2}\left(X_{2}\right)+f_{1}\left(X_{1}\right)$ while $f_{1}\left(X_{1}\right)^{f_{2}\left(X_{2}\right)}$ does not necessarily equate $f_{2}\left(X_{2}\right)^{f_{1}\left(X_{1}\right)}$. To put it differently, one does not, thanks to commuativity of addition, need to worry about the order in which one arranges variables in his model. This assumption of addition, as is illustrated, is taken as the core intuitive knowledge of regression as well as a great many other scientific models. It is consistent with the misunderstood principle of the razor insofar as it manages to "keep regression as simple as possible" in the sense of mathematical easiness, although admittedly it does circumvent unnecessary errors, in our case of (9), that might be given rise by other mathematical operations, for example, multiplication. If model (9) is alternatively speculated as

$$
Y=f_{1}\left(X_{1}\right) f_{2}\left(X_{2}\right)+\varepsilon
$$

then in a case where negative impact by both independent variables $X_{1}$ and $X_{2}$ jointing into a magnified positive impact upon $Y$, then it would be a major mistake.

There are notwithstanding other operations that may under specific problems superior than addition. The previous mentioned example of $f_{1}\left(X_{1}\right)^{f_{2}\left(X_{2}\right)}$ could have been model of better realistic merit in modeling problem of influence of interest rate and catastrophe events upon stock market indices for taking chronological fact into account. One should also be aware that operations that might fit into a situation should not confine to those already in existence. Or, it could be not only unary, binary, and functional operations, but also algebraic method that is not yet invented but might come into exist, let's abstractly denote it "input ${ }_{1}$ ? input ${ }_{2}$ ", in the future.

\subsection{De-Generalized Regression Model}

It is readily seen that the generalization of simple regression model $Y=b_{0}+b_{1} X_{1}+\varepsilon$ to its multivariate version

$$
Y=b_{0}+b_{1} X_{1}+b_{2} X_{2}+\cdots+b_{n} X_{n}+\varepsilon
$$

is a progress of naïve induction under the unjustified assumption of addition. Problems with this inductive generalization, as is illustrated throughout this paper, are first, a mistakable approach of cowardly resorting lack of knowledge to randomness; second, an overlook of a presumed major assumption of taking a mathematical operation for granted. To show ultimately the power of these assumptions, I shall try to propose a rough idea of what a robust model immune to naïve inductive generalization looks like.

For our analysis does not confine to regression model linear in parameter, it is preferable to present parameters as functions $f_{i}\left(b_{i}\right)$. But due to clarification of denotation, we shall distinguish function of parameter and function of variable so that $X_{i}$ in the model appears to be $F_{i}\left(X_{i}\right)$, hence we write

$$
\begin{aligned}
& Y=?_{0} f_{0}\left(b_{0}\right) ?_{1(p)} f_{1}\left(b_{1}\right) ?_{1(v)} F_{1}\left(X_{1}\right) ?_{2(p)} f_{2}\left(b_{2}\right) ?_{2(v)} \\
& F_{2}\left(X_{2}\right) ?_{3(p)} \cdots \cdots ?_{n(p)} f_{n}\left(b_{n}\right) ?_{n(v)} F_{n}\left(X_{n}\right) ?_{n} \\
& \left.\operatorname{Err}\left\{\left\{y_{i^{C}}, x_{i}\right\}\right\} ;\left\{X_{i}^{C}\right\}\right\}
\end{aligned}
$$

where $Y=$ explained variable,

$X_{1}, \cdots, X_{n}=$ assumed explanatory variables,

the question mark $?_{i(p)}=$ the $i^{\text {th }}$ mathematical operation, invented or uninvented, before parameter

$?_{i(v)}=$ the $i^{\text {th }}$ mathematical operation, invented or uninvented, before variable

$?_{0}$ at the beginning of the expression is installed to avoid the paradox that the equation itself is begin with a " $0+$ "

$\left\{y_{i^{C}}, x_{i}\right\}=$ complementary of obtained data, standing for incompleteness, given finitude of data

$\left\{X_{i}{ }^{C}\right\}=$ complementary of $\left\{X_{1}, \cdots, X_{n}\right\}$, other variables that have explanatory force but not presumed as explanatory variables in the regression model, given finitude of variables

At the cost of suffering from grand vagueness and major decrease in computational convenience, Model (12) eradicates a) naïvete of assuming addition, b) two kinds of error mentioned in Part II on the error term. However, careful inspection would make you find that model (12) simply replace "+ " with " $?_{i(p)}$ " and " $x$ " with " $?_{i(v)}$ " in expression (11), thus although it no longer assumes simple operation of addition and multiplication of elements, it is still not free from the assumption of addition unless it takes into account impact of a) autocorrelation of variables, and b) chronological order of variables, or in its essence, significance weight of variables.

Improvement a) could be achieved by condition a variable upon precedent one(s), rewrite equation (12) into

$$
\begin{aligned}
& Y=?_{0} f_{0}\left(b_{0}\right) ?_{1(p)} f_{1}\left(\left.b_{1}\right|_{b_{0}=b_{0}}\right) ?_{1(v)} F_{1}\left(X_{1}\right) ?_{2(p)} \\
& f_{2}\left(\left.b_{2}\right|_{b_{0}=b_{0}, b_{1}=b_{1}}\right) ?_{2(v)} F_{2}\left(X_{2} \mid X_{1}=x_{1}\right) ?_{3(p)} \cdots \\
& \cdots ?_{n(p)} f_{n}\left(\left.b_{n}\right|_{b_{0}=b_{0}, \ldots, b_{n-1}=b_{n-1}}\right) ?_{n(v)} \\
& F_{n}\left(X_{n} \mid X_{1}=x_{1}, \cdots, X_{n-1}=x_{n-1}\right) ?_{n} \operatorname{Err}\left\{\left\{y_{i^{C}}, X_{i} C\right\} ;\left\{X_{i}^{C}\right\}\right\}
\end{aligned}
$$


However, to assume model (13) it is necessary to assume a priori of $b_{i}$ (unlike distribution of $F_{i}\left(X_{i}\right)$, which there is possibility to be obtained by, for example, autoregressive technique). This can be avoided by incorporate $b_{i}$ into operation that is able to take into account parametrical information of $b_{i-1}$ so that equation (13) is transformed into

$$
\begin{aligned}
& Y=?_{b_{0}} ?_{b_{1}} F_{1}\left(X_{1}\right) ?_{b_{2}} F_{2}\left(X_{2} \mid X_{1}=x_{1}\right) ?_{b_{3}} \cdots \\
& \cdots ?_{b_{n}} F_{n}\left(X_{n} \mid X_{1}=x_{1}, \cdots, X_{n-1}=x_{n-1}\right) ?_{n} \\
& \operatorname{Err}\left\{\left\{y_{i^{C}}, X_{i} C\right\} ;\left\{X_{i}^{C}\right\}\right\}
\end{aligned}
$$

Here the original operation $?_{0}$ is dropped for the equation now starts alternatively with a $?_{b_{0}}$ operation that concerns the first parameter.

Some may argue that improvement $b$ ) is unnecessary for significance of impact of different explanatory variables would be well weighted by parameters they correspond to; for example, in a simple regression model of $Y=15+0.3 X_{1}+27 X_{2}+\varepsilon, \quad X_{2}$ is weighted magnificently more than $X_{1}$ in the sense that slope parameter of $X_{2}$ is of excessively greater value than that of $X_{1}$. This line of reasoning ignores the fact that it is the essence of the operation of addition, not the magnitude of parameters, that weights uniformly every one of its inputs. In the arbitrary simple regression model shown above, thanks to the method of addition, elements $0.3 X_{1}$, $27 X_{2}$, and the disturbance $\varepsilon$ are weighted averagely. Therefore, we shall expect elimination of uniformity of weights to be ability of operation rather than parameters. Note that any effort to attempt to incorporate equivalent of Equation (8)

$$
\operatorname{Err}\left\{\left\{y_{i^{C}}, x_{i^{C}}\right\} ;\left\{X_{i}^{C}\right\}\right\}=Y-b_{0}-\sum_{i=1}^{n} b_{i} X_{i}
$$

into Equation (14) as a rough method of decomposing would be futile unless, when it comes to estimate parameter vector $\left\{b_{0}, b_{1}, \cdots, b_{n}\right\}$, one employs technique other than least squares which is based on a bunch of assumptions of error term $\varepsilon$.

\subsection{Discussion}

Models (13) and (14) that of murderous vagueness and complex, partly due to uncertain mathematical operations, reveal in comparison to Equation (11) how much has been assumed by simple regression theory. First, regression model cowardly resorts lack of knowledge to normality for computational merits. This pseudo-randomness embraces, I believe, Aristotelian doctrine of natural places, or mean reversal, and could scarcely be backed by central limit theorem. I have shown that the assumption of normality of the disturbance may also stem from confusing functional-relationship model with regression model, the former backed by indubitable theorems and the letter by nothing but arbitrary speculation. Second, the idea of inductive generalization has been core of regression modeling. It assumes the mathematical operation of adding elements up without testing validity of dumping other methods, and most critically, it overlooks the possibility of invention of superior operations in the future. Complexity of Equation (13) and (14) is evidence for convenience provided by another assumption of regression model that explanatory variables are uncorrelated and chronologically independent.

\section{Conclusions}

It is now to realize that basic idea of modeling has actually run counter to what is suggested by Occam's Razor because in "keeping models as simple as possible" one needs to assume the most. It is only by truncating robustness of theories that they earn higher computability, and it is for their possibility of materialization that theories get publication. The question is, shall we criticize a method for its fragility if there is so far no better one available? One way of saving the dispute is to refuse to make use of any naïve approach in the first place, though naïvete usually is not detected until hindsight.

\section{References}

[1] K. R. Popper, "The Open Society and Its Enemies, Volume Two: Hegel and Marx," Routledge Classics, New York, 1945.

[2] K. R. Popper, "The Poverty of Historicism," Routledge Classics, New York, 1988.

[3] N. N. Taleb, "The Bed of Procrustes: Philosophical and Practical Aphorisms," Random House, 2010.

[4] D. N. Gujarati, "Essentials of Econometrics," 2nd Edition, Mcgraw-Hill College, New York, 1998.

[5] W. S. Robinson, "Ecological Correlations and the Behavior of Individuals," American Sociological Review, Vol. 15, No. 3, 1950, pp. 351-357. doi:org/10.2307/2087176

[6] L. Goodman, "Ecological Regression and the Behavior of Individuals," American Sociological Review, XVIII, Vol. 18, 1953, pp. 663-664.

[7] A. Lichtman, "Correlation, Regression, and the Ecological Fallacy: A Critique," The Journal of Interdisciplinary History, Vol. 4, No.3, 1974, pp. 417-433. doi:org/10.2307/202485

[8] F. E. Kydland and E. C. Prescott, "The Computational Experiment: An Econometric Tool," Journal of Economic Perspectives, Vol. 10, No. 1, 1996, pp. 69-85. doi:org/10.1257/jep.10.1.69

[9] Smets, Frank and R. Wouters, "An Estimated Dynamic Stochastic General Equilibrium Model of the Euro Area," 
Journal of the European Economic Association, Vol. 1, No. 5, 2003, pp. 1123-1175.

doi:org/10.1162/154247603770383415
[10] C. A. Sims, "But Economics Is Not an Experimental Science," Journal of Economic Perspectives, Vol. 24, No. 2, pp. 59-68. doi:org/10.1257/jep.24.2.59 